\title{
CXCR7 Transactivates and Triggers Signaling through EGFR
}

\author{
Georgios Kallifatidis \\ Department of Urology, University of Miami-Miller School of Medicine, USA
}

Corresponding author: Georgios Kallifatidis, Department of Urology, University of Miami-Miller School of Medicine, Gautier Research Building, Miami, FL 33136, Tel: 305-243-1017; E-mail: g.kallifatidis@med.miami.edu

Received date: March 12, 2016; Accepted date: March 29, 2016; Published date: April 02, 2016

Citation: Kallifatidis G. CXCR7 Transactivates and Triggers Signaling through EGFR. J Neoplasm 2016, 1:1

\section{Summary}

Over expression of chemokine receptors such as CXCR7 is a common hallmark of various cancers. Recent evidence suggests that CXCR7 interacts with the epidermal growth factor receptor (EGFR) to activate downstream mitogenic signaling and proliferation in tumour cells. Few current therapies directly target CXCR7; however, targeting the interaction between CXCR7 and EGFR might be a promising therapeutic strategy to inhibit proliferation in cancer cells.

Recent evidence suggests a significant contribution of chemokine receptor signaling in tumor growth, survival after chemotherapy, and organ specific metastasis [1]. Chemokine receptors are members of the seven trans-membrane guanine nucleotide-binding protein (G protein)-coupled receptor (GPCRs) superfamily, which is the most diverse class of cellsurface receptors. GPCRs mediate the actions of various hormones, neurotransmitters, and sensory stimuli by activating heterotrimeric G-proteins which bind on intracellular loop epitopes at the carboxyl-terminal tail of these receptors [2]. The CXC-chemokine receptor 7 (CXCR7) is a unique receptor that can be engaged by the chemokine's CXCL11 and CXCL12/SDF-1 [3] but unlike other classical chemokine receptors, it preferentially signals through the $\beta$ arrestin-2 pathway upon binding to its ligands $[4,5]$. $\beta$ arrestins are multifunctional adaptor/scaffold proteins which are ubiquitously expressed in most tissues $[6,7]$. $\beta$-arrestins are well known negative regulators of GPCR signaling that regulate desensitization. Once $\beta$-arrestins binds to GPCRs they uncouple receptors from heterotrimeric $G$ proteins and target them to clathrin coated pits for endocytosis. However, $\beta$ arrestins may serve as scaffolds to assemble multi-protein complexes that leading to downstream G-protein independent signaling events including ERK1/2 activation [2].

Interactions between GPCRs and receptor tyrosine kinases (RTKs) such as EGFR play a role in activation of ERK and thus cell proliferation $[2,8]$. Recent evidence demonstrates that $\beta$ arrestins function as signal transducers linking GPCRs to ERK1/2 signaling by either scaffolding members of the ERK1/2 cascade or by trans-activating RTKs [9]. Moreover, $\beta$-arrestin 2 was shown to regulate interaction of CXCR7 with EGFR in breast cancer cells where overexpression of $\beta$-arrestin 2 resulted in an increased phosphorylation of EGFR at Y1110 and promoted downstream ERK1/2 signaling and proliferation. Consistently, down regulation of CXCR7 in these cells resulted in a decrease of ERK1/2 and EGFR-Y1110 phosphorylation/ activation. Furthermore, downregulation of $\beta$-arrestin 2 resulted in a decrease in CXCR7-EGFR colocalization confirming a potential role of $\beta$-arrestin 2 in the interaction of EGFR and CXCR7. These observations suggest that $\beta$-arrestin 2 mediates CXCR7-EGFR crosstalk and plays a key role in activation of EGFR and downstream ERK signaling to mediate tumor cell proliferation [2].

More than $40 \%$ of currently marketed drugs modulate GPCR signaling [9]; however, few anti-cancer agents directly target GPCRs. Several approaches can be utilized to inhibit GPCR signaling; for example, molecules which antagonize the interaction of the ligand with the receptor, and antibodies that mask the agonist binding site [8]. However, new novel therapeutic strategies should employ relevant C-terminus inhibitors of CXCR7 that might interfere with the CXCR7-EGFR interaction. Targeting the interaction of CXCR7 and EGFR might be a first line therapy for patients presenting CXCR7 over expressing tumors. Since $\beta$-arrestin 2 regulates the interaction of CXCR7 and EGFR, targeting this scaffold protein and its binding to CXCR7 might also have relevant clinical significance.

In conclusion, a promising approach to inhibit the promalignance effects of GPCRs is to directly target their immediate downstream interactors such $G$ protein or $\beta$ arrestins. Inhibition of down steam effectors would broaden the effectiveness of a drug since multiple GPCRs signal through a much smaller number of effectors [8]. Since interaction of CXCR7 with EGFR seems to promote proliferation of breast and prostate cancers, targeting effectors that mediate the interaction between EGFR and CXCR7 might be of significant clinical relevance.

\section{References}

1. Salazar N, Castellan M, Shirodkar SS, Lokeshwar BL (2013) Chemokines and chemokine receptors as promoters of prostate cancer growth and progression. Crit Rev Eukaryot Gene Expr 23: 77-91.

2. Salazar N, Muñoz D, Kallifatidis $G$, Singh RK, Jordà $M$, et al. (2014) The chemokine receptor CXCR7 interacts with EGFR to promote breast cancer cell proliferation. Mol Cancer 13: 198.

3. Sánchez-Martín L, Sánchez-Mateos P, Cabañas C (2013) CXCR7 impact on CXCL12 biology and disease. Trends Mol Med 19: 12-22. 
4. Singh RK, Lokeshwar BL (2011) The IL-8-regulated chemokine receptor CXCR7 stimulates EGFR signaling to promote prostate cancer growth. Cancer Res 71: 3268-3277.

5. Rajagopal S, Kim J, Ahn S, Craig S, Lam CM, et al. (2010) Betaarrestin-but not $\mathrm{G}$ protein-mediated signaling by the "decoy" receptor CXCR7. Proc Natl Acad Sci USA 107: 628-632.

6. Ma L, Pei G (2007) Beta-arrestin signaling and regulation of transcription. J Cell Sci 120: 213-218.
7. Buchanan FG, DuBois RN (2006) Emerging roles of betaarrestins. Cell Cycle 5: 2060-2063.

8. Innamorati G, Valenti MT, Giovinazzo F, Carbonare LD, Parenti $M$, et al. (2011) Molecular approaches to target GPCRs in cancer therapy. Pharmaceuticals 4: 567-589.

9. Mancini A, Frauli M, Breton B (2015) Exploring the technology landscape of 7TMR drug signaling profiling. Curr Top Med Chem 15: 2528-2542. 